\title{
TRANSCENDENTAL FUTURE - IS IT A HEALTHY BELIEF OR A TIME PERSPECTIVE? THE TRANSCENDENTAL-FUTURE TIME PERSPECTIVE INVENTORY (TTPI) IN ESTONIAN
}

\author{
Riin Seema ${ }^{1,2}$, Anna Sircova ${ }^{3}$, and Arno Baltin ${ }^{2}$ \\ ${ }^{1}$ Tallinn School of Economics, ${ }^{2}$ Tallinn University, and ${ }^{3}$ Umea University
}

\begin{abstract}
This article assesses whether the second version of the Transcendental-future Time Perspective Inventory (TTPI; Zimbardo and Boyd 2008) measures a time perspective or a belief and how it is related to mental health in a highly secular country. The TTPI adapted into the Estonian language is described. Study 1 reports the results of validation performed on a large sample of adult students at different levels of education. Study 2 was carried out in a clinical sample with people turning to psychotherapy. Females scored significantly higher on TTPI than males, other demographic variables (age, level of education) were not related. TTPI scores were not related to mindfulness, self-esteem, wellbeing and depression. The TTPI is a reliable scale measuring belief in an afterlife, but not a time perspective. The TTPI items should ask how often people imagine themselves in a transcendental future context and if these thoughts are positive or negative.
\end{abstract}

Keywords: transcendental future belief, time perspective, mental time travel, subjective well-being, self-esteem, spirituality

DOI: $10.3176 /$ tr.2014.1.04

\section{Introduction}

At a certain point in anthropological time the human brain had developed to the level that people became aware of time and of their own existence (Allik and Tulving 2003). Together with the ability to imagine one's future a new kind of mental stress also appeared - awareness of the inevitability of death (Suddendorf and Corballis 2007). To allay this stressor, our early ancestors came up with a myth - a belief that death must be survivable (Newberg et al. 2001). Today the bigger part of people's beliefs has been passed on to them by their ancestors through religion or philosophy (Giovannoli 2001). 
According to Boyd and Zimbardo (2006) immortality is a vital issue for many people and therefore it is important to study people's after death beliefs. They called the time that extends from the death of the physical body to eternity transcendental future. Boyd and Zimbardo (2006) also claimed that the way people relate to the transcendental future is a time perspective (TP). Time perspective is a psychological construct that reflects people's attitudes, beliefs, and values related to time. TP describes whether people spend more time on thinking about the past, present or future and whether their thoughts are positive or negative in this process (Zimbardo and Boyd 2008:52). Over-reliance on particular temporal frames is learned from one's culture, religion, social class and family. Although time perspectives may depend on circumstances, they are still considerably permanent traits (Zimbardo and Boyd 1999).

Zimbardo Time Perspectives Inventory (ZTPI; Zimbardo and Boyd 1999) measures a time perspective profile, and consists of five subscales: Past Positive (PP), Past Negative (PN), Present Hedonistic (PH), Present Fatalistic (PF) and Future (F). Several studies have shown that time perspective is connected with self-esteem, subjective well-being, levels of depression, and mindfulness. Time perspective and self-esteem research has shown that the Past Positive and Future perspectives are connected with a high self-esteem, whereas the Past Negative and Present Fatalistic perspectives with a low self-esteem (Zimbardo and Boyd 1999, Sircova et al. 2008, Anagnostopoulos and Griva 2012). No relationship was shown between the Present Hedonistic perspective and self-esteem (Zimbardo and Boyd 1999).

A strong negative link was found between subjective well-being and the Past Negative time perspective (Boniwell 2005, Drake et al. 2008, Sircova and Mitina 2008, Boniwell et al. 2010). Subjective well-being and both the Past Positive and Present Hedonistic time perspective were found to be positively correlated (Drake et al. 2008, Boniwell et al. 2010, Zhang and Howell 2011). The Future perspective was not related to well-being (Drake et al. 2008, Boniwell et al. 2010). At the same time Romero and Uomoto (2009) found that the Future and Present Fatalistic perspectives were predictors of lower well-being and the Present Hedonistic perspective did not predict higher positive well-being.

As investigations have shown, the Past Negative perspective is correlated with depression (Zimbardo and Boyd 1999, Roseanu et al. 2008, Sircova et al. 2008, Anagnostopoulos and Griva 2012). Positive perception of the past was inversely correlated with depression (Zimbardo and Boyd 1999, Anagnostopoulos and Griva 2012), as was the Future time perspective (Zimbardo and Boyd 1999, Anagnostopoulos and Griva 2011). Individuals with the Present Fatalistic perspective (Zimbardo and Boyd 1999, Roseanu et al. 2008, Anagnostopoulos and Griva 2012) and Present Hedonistic perspective (Roseanu et al. 2008) have more psychological difficulties and are more frequently exposed to depression. Drake et al. (2008) found that mindful attention and awareness correlated positively with Past Positive and negatively with Past Negative, Present Hedonistic, and Present 
Fatalistic traits. In their study the relationship between Future and MAAS was positive but not significant.

Boyd and Zimbardo (2006) stated that as a psychological construct the transcendental future time perspective exhibits attributes similar to those associated with the more conventional past, present and future time perspectives. Boyd and Zimbardo (2006) devised a scale - the Transcendental-future Time Perspective Inventory (TTPI) that reflects an ecumenical belief in the existence of life after death. Meanwhile, Boyd and Zimbardo (2006) verified that the Transcendentalfuture time perspective does not correlate considerably with the other time perspectives suggested by them. Their study did not extend to how thoughts about transcendental future are related to mental health indicators. Later Zimbardo and Boyd (2008) made changes into the TTPI scale to integrate it with the time perspective theory. They used the same introduction and scale as in the Zimbardo Time Perspective Inventory (ZTPI, Zimbardo and Boyd 1999).

So far no studies with the TTPI and mental health variables have been performed. We were interested in how thoughts about transcendental future assessed with the TTPI are connected to well-being indicators in Estonia, a small European country with religious freedom, where religion plays an important role only in the life of a minority of the population (Annual Report of Religious Freedom 2010). The aim of the current study was to test if the second version of the TTPI (Zimbardo and Boyd 2008) measures the time perspective construct as theoretically defined. Our aim was to explore if transcendental future measured with the TTPI is a time perspective, a belief or both. Therefore, we set out to adapt and validate the Transcendental-future Time Perspective Inventory (TTPI) (Zimbardo and Boyd 2008) into the Estonian language, which does not have future grammar (will), and differs in this sense form English. In the literature review the current article introduces the human ability to think about and to imagine a transcendental future, as well as a belief in transcendental future and its relation to subjective well-being.

\subsection{The ability to imagine the transcendental future}

Time, either the past or future does not necessarily physically exist in real life, but it is present in the mental or neurocognitive reality of a human being (Tulving and Szpunar 2012).

As the past was once present, but the future is just a fiction, we can think that different brain mechanisms are behind remembering the past and imaging the future. In fact, when people think about past or future, the same semantic and episodic memory (prospection) systems cooperate (Suddendorf and Corballis 2007). Semantic memory and semantic or noetic awareness is used when people think about the past or future and do not remember or imagine it (Tulving 2002). Semantic memory includes encoded, but tenseless facts and ideas about oneself and the common facts of the world (Vandekerckhovea and Panksepp 2011). Episodic memory together with autonoetic consciousness (Tulving 2002) make it 
possible to retrieve or recall details of events that have happened to a person and also enables one's episodic prospection (Suddendorf and Corballis 2007).

The ability to 'travel' back and forth in time to own subjective past, present or future experiences connected with neurocognitive processes described above has been called chronesthesia (Tulving 2002). Although language is not indispensable for mental time travel, it provides the clearest evidence of it (Suddendorf and Corballis 2007). However, the sense of mental time travel by itself does not mean the recollection is accurate, it merely implies that autonoetic awareness circuits are involved in the activation of stored or internally generated representations (Siegel 2012). 'Mental time travel' enables people to imagine endless amounts of various points and options on the space-time continuum even from the time before their birth or after their death (Tulving and Kim 2007, D'Argembeau et al. 2010).

Consequently all healthy people are able to think about a transcendental future. TP theory (Zimbardo and Boyd 2008) leads us to ask the questions how mental health is related to what and how often people think about the time after their death. From the socio-psychological perspective it does not matter what actually happens after we die. Like Zimbardo and Boyd (2008), we are interested how beliefs about life after death affect people in the present.

\subsection{Transcendental future beliefs}

As it is not directly possible to ascertain the possibility of an afterlife, all claims about one's future, also beliefs about death being the end of existence - are ultimately matters of belief (Burris and Bailey 2009). But it is the nature of a strong belief to accept the true value of a particular mental content without the need for an immediate proof, or for any proof (Damasio 2001).

Belief is a result of intuition meaning that the processing mechanisms underlying belief are partly unconscious (Damasio 2001). Most of the time people are unaware of their beliefs (Westbery and Dennett 2000). Belief is like a disposition, an aptitude for certain behaviors in certain situations (Villoro 1998).

\subsection{Transcendental future - a healthy belief?}

In psychology, a religious belief is not considered pathological, if a large number of people believe in it (Gollnick 2005). It must be stressed, though, that a transcendental future is a component of many religious beliefs, but not their synonym (Boyd and Zimbardo 2006). Little research is available specifically on the afterlife and thus making it hard for generalizations about the connection between the afterlife beliefs and mental health (Flannelly et al. 2008). Most researchers have looked into the belief in an afterlife as being connected with fear of death (Rose and O'Sullivan 2002) or bereavement (Clarke et al. 2003) and the majority of research has been conducted with the sample population limited to elderly citizens or the severely ill (McClain-Jacobson et al. 2004, Elahi 2008).

Still, Flannelly et al. (2006) detected a statistically significant weak inverse relationship between belief in life after death and six measures of psychiatric symptomology. Flannelly et al. (2008) studied the connection between both the 
positive and negative afterlife beliefs and psychiatric symptoms. They discovered that negative beliefs were related with weaker mental health. We can conclude that the connection between afterlife beliefs and mental health is mixed and dependent on which specific beliefs are under investigation (Galek and Porter 2010).

As so far this topic has been almost taboo in academic psychology (Boyd and Zimbardo 2008), this has not been studied in Estonia. In our study we further explore this connection between a person's beliefs in life after death and mental health indicators: self-esteem, subjective well-being, depression and mindfulness in a country with unique religious and spiritual culture.

\subsection{Religion and spirituality in Estonia}

Young Estonians are one of the least religious groups in Europe (Kairos Future 2007). Most schools do not teach religion among their subjects (Schihalejev 2010). At the same time, the majority of Estonians (54\%) believe that there is some spirit or life force and less than one fifth (16\%) declare that they believe in God (Special Eurobarometer 2005). Estonians are highly secular (Schihalejev 2010).

The secular beliefs of Estonians today are quite similar to nature-worship and several ancient customs are still being observed (Kaasik 2004). The Estonian ancestors were Finno-Ugrians and practiced a religion that could be called the religion of the forest (Gawthrop 1999) or the earth-centered religion (Kaasik 2004). They believed that all plants, animals, and other living creatures have a soul (Gawtrop 1999).

The notion of spirituality has a more personal and psychological context, whereas the notion of religion is more institutional and sociological (Hood 2009). We did not ask about participant's religiosity as the majority of population is not associated with any religion (Schihalejev 2010). We considered the TTPI as a scale to measure the transcendental future related spiritual belief (e.g. "I believe in spirits"). Next we describe the adaptation process of the TTPI into the Estonian language and cultural context.

\section{Methods}

\subsection{Process of adaptation}

The adaptation of the TTPI took place in 2009-2010 as a part of a larger project. The scale was translated into Estonian by four linguists and two authors of the current article and back-translated by two separate linguists. Experts' meetings followed to compare the translations, back-translations and original and to adapt the questionnaire. The scale was reviewed by an Estonian philologist.

\subsection{Pilot studies}

Three pilot studies (Ns $=32,42$ and 43) were conducted. Group and individual interviews were carried out. In addition to filling in the questionnaires, the 
respondents were asked to give feedback on the comprehensibility of the items and their linguistic correctness. Reliant on the respondents' feedback, modifications were made to the questionnaires after every pilot study.

\subsection{Study 1}

The objective of the first study was to explore the factor structure and reliability of the TTPI in Estonian adult student's sample. The aim was to determine the level of belief in a transcendental future among Estonian students with different levels of education and how that belief is connected with well-being indicators.

\subsection{Sample}

The study sample included a total of 668 students from different places in Estonia of whom 367 (55\%) were in higher education, 205 (30.5\%) in professional higher education, $86(13 \%)$ in vocational education and $10(1.5 \%)$ who did not state their educational background. $510(76.2 \%)$ respondents were female and 158 (23.8\%) were male.

The self-report measures were administered primarily through an internet survey. Some participants completed the measures on paper during class time.

\subsection{Measures}

The adapted Transcendental-future Time Perspective Inventory (TTPI; Zimbardo and Boyd 2008). In the English introduction of the TTPI (Zimbardo and Boyd 2008) the respondent was asked to answer the question: "How characteristic or true is this of you?" Based on our pilot studies the Estonian TTPI asks "How true do these statements appear to you?" (Kuivõrd õiged need väited Sinu arvates on). The Estonian scale measures beliefs in transcendental future or time after death on a five point Likert scale (very untrue - very true). The Estonian scale has 9 items, compared to the original with 10 items. Based on the results of the pilot studies item \#2 from the original scale, "My body is just a temporary home for the real me", was reformulated as "A person has an immortal soul", as several students found the original item difficult to understand. The item \#5 "The theory of evolution adequately explains how humans came to be" was excluded from Estonian version based on the EFA and CFA results. The internal consistency in this sample was, $\alpha=.86$.

The adapted Zimbardo Time Perspective Inventory (ZTPI; Zimbardo and Boyd 1999), adapted by Seema and Sircova (submitted). The Estonian scale measures a person's orientation and attitudes towards time on a five point Likert scale (very untrue - very true). The Estonian scale has 25 items; five items in every subscale.

Past Negative (PN) is a tendency to think about the past with pain and regret e.g. "Painful past experiences keep being replayed in my mind" (sample $\alpha=.83$ ). Past Positive (PP) is a tendency to have positive and nostalgic thoughts and feelings about the past e.g. "It gives me pleasure to think about my past" (sample 
$\alpha=.69)$. Present Hedonistic (PH) is a tendency to only concentrate on the present, get satisfaction in the present without worrying about future results e.g. "I take risks to put excitement in my life" (sample $\alpha=.73$ ). Present Fatalistic (PF) tends to perceive the present with hopelessness and with the feeling of incapability, because both the present and future seem to be predestined e. g. "My life path is controlled by forces I cannot influence" (sample $\alpha=.67$ ). Future $(F)$ is a tendency to think and act according to a plan in order to get rewarded in the future e.g. "When I want to achieve something, I set goals and consider specific means for reaching those goals" (sample $\alpha=.75$ ).

Mindful Attention Awareness Scale (MAAS; Brown and Ryan 2003) adapted by Seema, Brown, Sircova, Quaglia, Konstabel, and Baltin (submitted). The scale measures open or receptive awareness and attention to what is taking place in the present (Brown and Ryan 2003). The adapted, 15-item MAAS measures trait mindfulness indirectly, as does the original scale e.g. "It seems I am 'running on automatic', without much awareness of what I'm doing" (sample $\alpha=.86$ ).

Self-esteem The 10-item Estonian Rosenberg Self-Esteem Scale (ERSES; Pulmann and Allik 2000) determines overall evaluation of one's worth or value (Rosenberg 1989) on a 5-point scale ranging from strongly disagree to strongly agree. The internal consistency in this sample was, $\alpha=.88$.

Psychological well-being The WHO-Five Well-being Index (WHO- 5) (Sisask et al. 2008) measures positive mood, vitality, and interest in things with five statements on a 6-point scale (never to always). Higher values indicate higher psychological well-being (sample $\alpha=.87$ ).

\section{Results}

\subsection{Factorial structure of the Estonian TTPI}

The exploratory factor analysis (EFA) was first performed, using the maximum-likelihood method. The Kaiser-Meyer-Olkin measure of sampling adequacy value was .87 , and Bartlett's test of sphericity was significant, $\chi^{2}(2302)=45, p<.0001$. The scree plot indicated a clear 'elbow' between factors 1 and 2 , and thus a one-factor solution was applied. The extracted factor accounted for $43.7 \%$ of the variance. All items had factor loadings over .5 , except the original item \#5 that was excluded.

Confirmatory factor analysis. (CFA) was used to confirm the fit of the onefactor solution. CFA was performed on the second half of the randomly split sample $(\mathrm{n}=335)$ using maximum likelihood estimation with robust standard errors. The results indicated a good-to-acceptable fit ( $\mathrm{Hu}$ and Bentler 1999): Satorra-Bentler $\chi^{2}(21)=47.9 ; \chi^{2} /(\mathrm{df})=2.28 ; \mathrm{CFI}=.97$; RMSEA $=.062(09 \%$ CI $.039, .085)$. Error co-variances were added to the model and item \#4 was omitted in the course of the analysis. The item-by-item standardized factor loadings after CFA are reported in Table 1. 
Table 1. TTPI scale means (M), standard deviations (SD), EFA factor loadings (F), CFA standardized factor loadings (ZF). Adult students' sample

\begin{tabular}{|c|c|c|c|c|}
\hline & M & SD & $\mathrm{F}$ & $\mathrm{ZF}$ \\
\hline $\begin{array}{l}\text { 1. Only my physical body will ever die. Vaid minu } \\
\text { füüsiline keha on see, mis kunagi sureb. }\end{array}$ & 3.48 & 1.220 & .621 & .563 \\
\hline 2. Death is just a new beginning. Surm on üksnes uus algus. & 3.12 & 1.214 & .704 & .640 \\
\hline 3. I believe in miracles. Ma usun imedesse. & 3.66 & 1.238 & .743 & .618 \\
\hline $\begin{array}{l}\text { 4. The theory of evolution adequately explains how humans } \\
\text { came to be. Evolutsiooniteooria selgitab inimkonna } \\
\text { tekkimist piisavalt hästi. }\end{array}$ & 3.51 & 1.150 & -.348 & $\mathrm{n} / \mathrm{a}$ \\
\hline 5. Humans possess a soul. Inimesel on hing. & 4.34 & .912 & .725 & .679 \\
\hline $\begin{array}{l}\text { 6. Scientific laws cannot explain everything. Teadus ei } \\
\text { suuda kõike seletada }\end{array}$ & 4.22 & 1.079 & .595 & .522 \\
\hline $\begin{array}{l}\text { 7. I will be held accountable for my actions on earth when I } \\
\text { die. Kui ma suren, tuleb mul vastutust kanda oma elu } \\
\text { jooksul tehtud tegude eest. }\end{array}$ & 2.74 & 1.199 & 677 & .599 \\
\hline $\begin{array}{l}\text { 8. There are divine laws by which humans should live. On } \\
\text { olemas jumalikud seadused, mille järgi inimesed peaksid } \\
\text { elama. }\end{array}$ & 2.60 & 1.263 & .568 & .511 \\
\hline 9. I believe in spirits. Ma usun vaimude olemasolusse. & 3.48 & 1.302 & .724 & 689 \\
\hline 10. Humans have immortal soul. Inimesel on surematu hing. & 3.17 & 1.223 & .794 & .810 \\
\hline
\end{tabular}

\subsection{Descriptive statistics}

The mean score in this sample was 3.4, with a standard deviation of 0.8 . The minimum score was 1.0 and maximum 5.0.

\subsection{Validity of the TTPI}

The correlations between the TTPI with the ZTPI subscales, the WHO-Five Well-being Index, the Estonian Rosenberg Self-Esteem Scale, and the MAAS, are shown in Table 2. The TTPI and the Future, Past Positive and Present Fatalistic were slightly positively correlated as in the original Boyd and Zimbardo study (2006). The other measures did not correlate with the TTPI.

\subsection{Group differences in the TTPI scores}

To test for statistically significant differences in TTPI scores between gender, age, and education level groups, one-way ANOVA analysis was performed. The analysis showed that females scored significantly higher on TTPI than males $(\mathrm{F}(1,667)=35.57, \mathrm{p}>.0001)$. The TTPI scores did not differ among people from different age groups $(18-25,26-36$ and over 36 years) or among students at different educational levels, ps $>.05$.

To further assess the validity and reliability of the TTPI, we used Study 2 to examine these issues in a clinical sample. We tested for test-retest reliability both with general population and the clinical sample. 
Table 2. Pearson correlations between the TTPI with other scales in adult students' sample

\begin{tabular}{l|c|c|c|c}
\hline & TTPI & $\begin{array}{c}\text { RSES } \\
\text { self-esteem }\end{array}$ & $\begin{array}{c}\text { WHO-5 } \\
\text { well-being }\end{array}$ & MAAS \\
\hline Past Negative & .030 & $-.497(* *)$ & $-.316\left(^{* *}\right)$ & $-.339(* *)$ \\
Past Positive & $.233(* *)$ & $.178(* *)$ & $.194(* *)$ & .031 \\
Present Hedonistic & .069 & .067 & $.202(* *)$ & $-.132(* *)$ \\
Present Fatalistic & $.193(* *)$ & $-.265(* *)$ & $-.138(* *)$ & $-.226(* *)$ \\
Future & $.114(* *)$ & $.259(* *)$ & $-.316(* *)$ & $.253(* *)$ \\
MAAS mindfulness & -.054 & $.339(* *)$ & $.271(* *)$ & \\
WHO-5 well-being & .074 & $.566(* *)$ & & \\
RSES self-esteem & .026 & & & \\
\hline
\end{tabular}

Note: $\mathrm{N}=568$. ZTPI subscales: Past Negative, Past Positive, Present Hedonistic, Present Fatalistic, Future; WHO-Five Well-being Index (WHO- 5); Estonian Rosenberg Self-Esteem Scale (RSES); Mindful Attention Awareness Scale (MAAS).

${ }^{* *} \mathrm{p}<01$.

\section{Study 2}

The Tallinn Medical Research Ethics Committee granted the approval to conduct this study (application no 1022, resolution adopted 20 Dec. 2010). The aim was to explore the connection between transcendental future belief and wellbeing among individuals going through psychotherapy process. In addition we aimed to test the hypothesis that TTPI measures two components of time perspective - the bias to think about a transcendental future time and positive or negative attitude. Multidimensional scaling (MDS) was used to analyze the data and to explore similarities or dissimilarities in the data. It allows gaining insight in the underlying structure of relations between concepts, or variables in question (Van Deun and Deldeke 2000).

To test for a positive or negative affect we used well-being, self-esteem and depression scales. To test whether the TTPI measures awareness of transcendental future thoughts we used the Mindful Attention and Awareness Scale MAAS. Zimbardo and Boyd (2008) discuss that mindfulness is a type of time perspective as it has important components of time perspective - orientation to a specific time and an attitude. Mindfulness as a holistic present includes past and future in an extended state of focus on the present. Mindfulness operates as a witness on mental content (Brown and Holt 2011).

\subsection{Sample}

Sample for this study consisted of 49 people who enrolled in some kind of psychotherapy (e.g. Cognitive-Behavioral therapy, Psychodrama, Family therapy, NLP, Breathing therapy and Gestalt-therapy). These people addressed different issues in their psychotherapy: depression, anxiety, burn-out, woman's role, 
relationships, eating problems, trauma and self-growth. For treating emotional problems 11 people were taking medications. Forty five respondents were females and 4 were males. The average age was 41.1 years $(S D=10.6)$.

\subsection{Measures}

The same measures were used in this study as in Study 1. We added one more scale since participants were a clinical sample.

Depression Scale (DEPS; Salokangas et al. 1995) in the Estonian language (Aluoja et al. 1999) is a 10-item self-rating scale for depression screening. The symptoms of experiencing depression during the past month are rated on a 4-point scale (not at all - extremely). Sample $\alpha=.91$.

\section{Results}

\subsection{Validity of the TTPI in the clinical sample}

The TTPI was not significantly related with any of the TP subscales, the WHOFive Well-being Index, the Estonian Rosenberg Self-Esteem Scale, the MAAS and the DEPS Depression Scale. All ps >.05.

\subsection{Reliability}

The test-retest reliability of the Estonian version of the TTPI was assessed with 43 adult students and 11 psychotherapy clients over a 6-month period. The correlation was $.80, \mathrm{p}<.001$.

\subsection{Two underlying dimensions of the TTPI, the MAAS mindfulness and well-being scales}

With MDS we tested the hypothesis that the TTPI measures a time perspective - orientation towards a transcendental future time as well as an attitude (positive or negative), by making the data accessible to visual inspection and exploration in two-dimensional space. The closer the any two scales as points are together, the more positively they are correlated or the more similar they are (Borg and Groenen 2010). Euclidean distances from the standardized data were created. The analysis revealed two dimensions (see Fig 1).

Based on one dimension the TTPI measures a very different construct compared to mindfulness, or awareness of present thoughts and perceptions (Brown and Ryan 2003). We called this dimension 'aware or unaware of psychological time'. The second dimension is 'positive or negative affect' and the TTPI is hardly at all related with this dimension. 

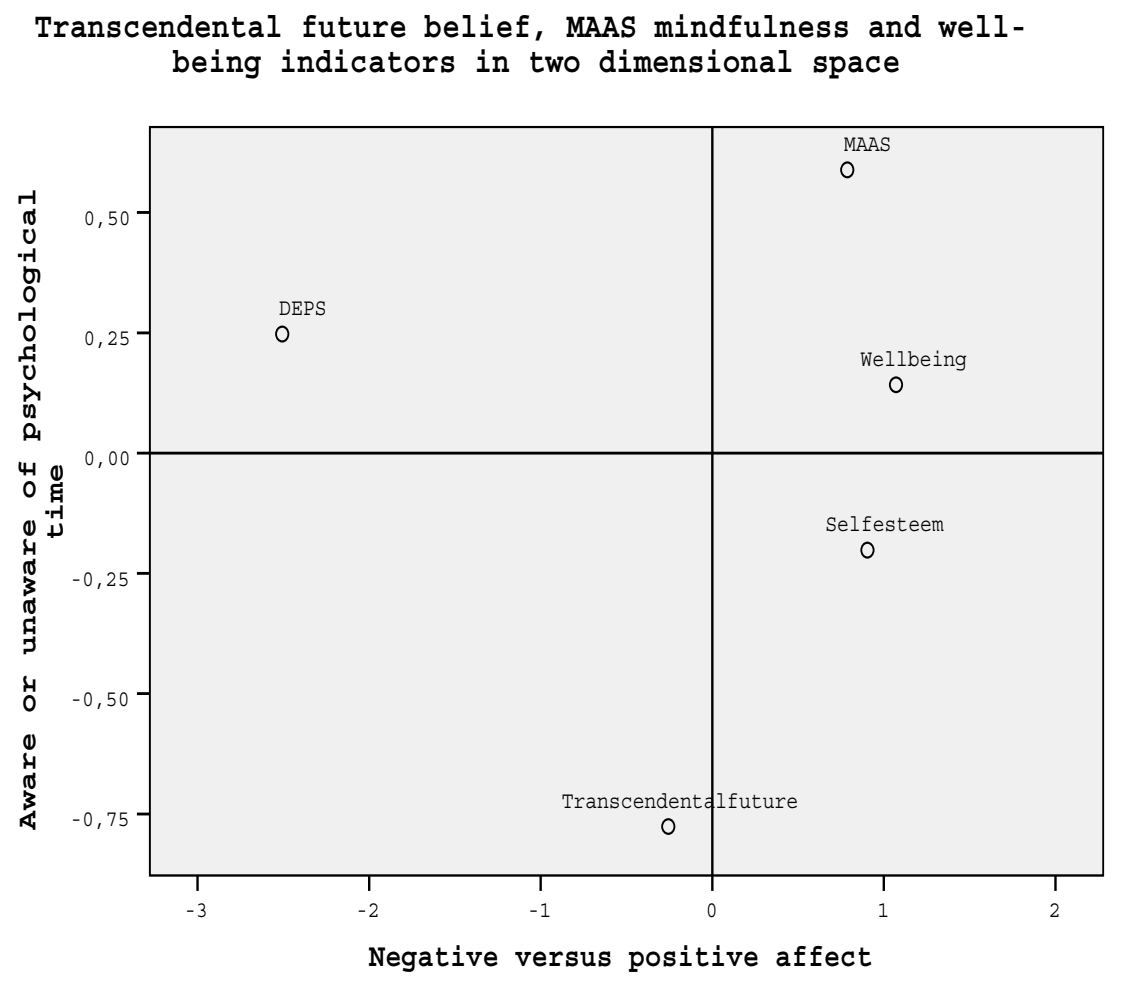

Figure 1. Euclidian distance model. Representation of (dis)similarities of the TTPI scale and wellbeing indicators in two-dimensional space. The scales are: RSES self-esteem, WHO-5 well-being, MAAS mindfulness, DEPS depression. For matrix Stress $=.03532$, RSQ $=.9954$.

\section{Discussion}

The aim of this study was to obtain a deeper understanding of the human experience of mental time travel, especially of the thinking and imagining about a transcendental future, or future after one's death. In the studies presented, we explored if the adapted Transcendental-future Time Perspective Inventory (Zimbardo and Boyd 2008) measures a time perspective or a belief and how its scores are related to mental health indicators in Estonia.

Theoretically, "mental time travel", as a way of consciously experiencing or imagining "subjective time" in a transcendental future, is possible (Tulving and Kim 2007, Nyberg et al. 2010, D'Argembeau et al. 2010). However, human beings are not able to ascertain the possibility of an afterlife; this is a matter of belief (Burris and Bailey 2009). Bennett and Hacker (2003) stated that it is important not to confuse the concept - which may be right or wrong, with the belief in a concept, which may be doubtful, certain, or even passionate. In our pilot studies, students were willing to discuss transcendental future even when they told that they do not 
believe in this time concept. Some students mentioned that they have never thought about this topic (spirituality or a transcendental future).

If we consider a transcendental future to be a time perspective (Zimbardo and Boyd 2008), as an individual differences variable, it should describe how often a person thinks about transcendental future and whether the thoughts are positive or negative in this process. It should reflect attitudes, beliefs, and values related to this subjective time.

In our studies, we examined (a) the factorial structure of the Estonian TTPI, (b) the reliability (internal consistency and repeatability) and validity both in adult and clinical samples, (c) how the construct of transcendental future maps into the time perspective coordinates and (d) how the scale scores vary by demographic groups (age, sex, level of education). As Estonian grammar related to time is different from English grammar, the authors of the current article became especially aware of the expressed temporality of the scale items in the adaptation process. Some culture and language specific modifications were introduced.

The research shows that the single-factor model was supported in the Estonian context. Both the internal consistency and repeatability of the scale were high and stable over time, a 6-month period. This shows that the items tend to be measuring the same 'thing' (Trull and Prinstein 2013). However, this does not in itself demonstrate that the TTPI items are actually measuring a time perspective - the bias to think positive or negative thoughts about a transcendental future. The existence of only one factor appeared problematic, as the TP construct has two past time perspectives (PP and $\mathrm{PN}$ ), and it would be logical that there are also two future and transcendental future perspectives. As Suddendorf and Corballis (2007) stated, when people think about past or future, the same semantic and episodic memory (prospection) systems cooperate. The original ZTPI scale has only one future subscale (F), but lately Carelli et al. (2011) added the Negative Future subscale (NF) to the Swedish ZTPI.

The Estonian cultural and religious background is reflected in the adapted scale. In the original TTPI (Boyd and Zimbardo 2008) the middle point of the scale is 'neutral'. Neutral means something is neither true nor false (Goldstein et al. 2005). In the Estonian version the middle point was changed to 'I do not know' based on pilot studies, as Estonians are highly secular or agnostic. The mean score of the TTPI in this sample was 3.4, that is a little above midpoint of the scale. The item 'Humans possess a soul' got the highest mean score 4.3, with the lowest standard deviation. Crick (1994) mentions that in spite of differences in religion, the majority of human beings agree that people have souls. At the same time people from different religions have a different opinion about what happens with the soul after the death of the body. Factor analysis showed that the reformulated item "Humans have an immortal soul" had the highest factor loading. The item expressed the measured latent trait well.

In the current research, the TTPI was related to the Past Positive perspective even more than in the original Boyd and Zimbardo (2006) study. Attitudes and beliefs related to time are learned from one's culture and family (Zimbardo and 
Boyd 1999). One item in the PP subscale is "I like family rituals and traditions that are regularly repeated". Despite the fact that Estonians are highly secular, they are profoundly attached to folk calendar customs merged with Christian holidays (O'Connor 2006). Those people who have positive memories about their past have probably internalized those beliefs and values. A slight positive link between the TTPI and the Present Fatalistic subscale can be explained with a belief in one's faith. The Estonian TTPI was also slightly related to the Future perspective.

In Estonian studies, the TTPI was not related to self-esteem, well-being and mindfulness.

The fact that time perspective constructs predict subjective well-being has been generally internationally confirmed (1st International Conference on Time Perspective 2012); therefore our findings raised questions about the validity of the TTPI. To further explore the issue, we tested whether this scale (Zimbardo and Boyd 2008) actually measures a time perspective; how often a person thinks about a specific time with a specific attitude. The multidimensional scaling analysis revealed that the TTPI items do not reflect positive or negative affect (see Figure 1). At the same time, the TTPI seems to measure a construct not related to or even somewhat opposite to mindfulness. A relative absence of mindfulness means an individual refuses to acknowledge or attend to a certain thought, emotion, motive or object of perception (Brown and Ryan 2004). This means that the TTPI does not measure how often a person thinks about a transcendental future. We can conclude that the TTPI scale measures beliefs that are usually unconscious. A person is conscious of a belief only while currently thinking about what is believed (Bennett and Hacker 2003). However, as the MDS analysis is a simplification, Giugere (2006) have stated that researchers should be careful when making strong interpretations based on this analysis.

One explanation why those beliefs that TTPI measure are separate from a person's affects (depression), evaluations of one-self (positive or negative), interests and does not fall into the focus of awareness is based on the face validity of the scale. We found that most of the items do not express either future grammar (will) or temporal adverbs. Only two items in the TTPI scale are about future time (e.g. "I will be held accountable for my actions on earth when I die"). The scale items measure mostly beliefs in certain transcendental future related ideas and facts (e.g. "Humans possess a soul") that represent the semantic memory system. The semantic memory system is by definition a "present-oriented" memory system which provides information of one's own past and the world detached from personally colored affective experiences (Vandekerckhove and Panksepp 2009, Vandekerckhovea and Panksepp 2011). These kinds of ideas are largely learned from the person's environment and religious background (Hood et al. 2009). The scale items should be connected to personal imaginings enriched by characteristics like temporality and affectivity (e.g. "I often imagine pleasurable situations that will take place in my afterlife", "I frequently picture that bad things are happening in my transcendental future"). They must be encoded in a greater 'depth' within 
the brain than semantic memories (Vandekerckhove and Panksepp 2009, Vandekerckhovea and Panksepp 2011).

The transcendental future time seems to be some kind of an extension of an individual's regular future. Burris and Bailey (2009) devised an after death beliefs scale that reflects episodic prospections; about what a person thinks will happen to their body, identity and consciousness after their death. They did not research the connection to mental health, but they found correlations between these imaginings and death concerns, reflecting the individual's important personal values. These kinds of beliefs were related to self-reported religion. At the same time, Flannelly et al. (2008) studied pleasant and unpleasant afterlife beliefs as related to psychiatric symptomology and found significant relationships in the predicted direction; however, all these correlations were weak, below .2. In their study only one item with mixed meaning, 'eternal reward/punishment', did not correlate with mental health - showing that transcendental future time matters for psychological health only if it has a special positive or negative meaning to a person. Neither of those studies asked how often a person contemplated about these after death thoughts.

The TTPI scale measures transcendental future related belief. In English the word belief is ambiguous; it means both psychological state or believing and the propositional content of that state (Bennett and Hacker 2003, Schellenberg 2005). In the adapted TTPI two items are about believing. Those items are "I believe in miracles" and "I believe in spirits". The rest of the items are about what is believed.

Zimbardo and Boyd (1999) stated that the TP reflects a person's orientation, attitudes, beliefs, and values related to a certain time frame and is related to psychological well-being. It seems that in order to measure the Transcendentalfuture TP is not enough to measure only beliefs, but orientation, attitudes and values also must be assessed. The scale should measure how often people think or imagine themselves in a transcendental future context and if these thoughts are positive or negative, based upon the personal meaning or value of these projections.

Researches have shown that belief in an afterlife is related to religiosity (Dezutter et al. 2008, Burris and Bailey 2009) and mental health (Flannelly et al. 2006, Flannelly et al. 2008). Since Estonia is a secular and agnostic society (Schihalejev 2010), transcendental future belief would not be expected to show a positive association with mental health indicators. We recruited also a clinical sample to participate in our study, but in a way, it was a 'death free' sample. We had no people with suicidal attempts, terminally ill, or having experienced a death of a significant other (parent, sibling, spouse, etc.), where the issues of attitudes towards death and coping with it are at stake.

Women have been observed to believe in transcendental future more strongly than men (Boyd and Zimbardo 2006, Ortuño et al. 2012). Our findings replicate these results. Age and the level of acquired education were not related with the 
transcendental future belief; this means that beliefs in life after death are equally represented across different groups of population in Estonia.

\section{Conclusion}

The present study shows some important features. First, we base our study not only on a large adult sample in Estonia, but also on a sample of psychotherapy clients. Second, we address a very complex phenomenon of belief in afterlife in a highly secular and agnostic society. With this study we provide a deeper understanding of the Transcendental-future time perspective.

In conclusion, the transcendental future is 'subjective time' that can be called a belief. Our research showed that people have firm ideas related to a transcendental future measured with the TTPI scale, but these beliefs are not related to indicators of subjective well-being among Estonians. Transcendental future could also be a time perspective - a personality trait that describes how often a person imagines one's afterlife with positive or negative attitude. However, Estonian TTPI does not measure this. In order to study time perspective, the scale items should reflect more mental time travel type of thinking, they should ask how often people think or imagine themselves in a transcendental future context and also if these thoughts are positive or negative. The TTPI is reliable, but not valid, as the scale is measuring a belief and not a time perspective. For this reason the name of the current TTPI scale is misleading. Still, finding an answer to the question "If, and what kind of, transcendental future TP-s are healthy?" is important with reference to mental health in different countries. This has not been studied before.

\section{Limitations and future research}

The samples consisted mostly of females and the psychotherapy sample was small. Future research should focus on devising the positive and negative transcendental future time perspective subscales. Cross-sectional as well as longitudinal studies would help to reveal the relationship between transcendental future belief, transcendental future time perspectives and their connections with mindfulness and mental health. The relationship between Transcendental-future belief, Transcendental-future time perspective, and religiosity should be studied.

\section{Acknowledgments}

We thank the consultants Dr. Philip G. Zimbardo, Mare Pork, Dr. Glenn Hogan, Andres Sild, Velli Parts, Pille Isat and Mare Vennik. We also thank the translators Külli Nõmme, Anne Leius and Estonian philologist Kristina Kask. 
Portions of this paper were presented at the 1st International Conference on Time Perspective, September 2012, Coimbra, Portugal.

\author{
Address: \\ Riin Seema \\ Tallinn School of Economics \\ Tammsaare tee 147 \\ 12915 Tallinn \\ Estonia
}

E-mail: riin@smail.ee

Tel.: +37255932253

\title{
References
}

Allik, J. and E. Tulving (2003) "Ajas rändamine ja kronesteesia". [Travelling in time and chronestesia.] Akadeemia (Tartu) 15, 915-937.

Anagnostopoulos, F. and F. Griva (2012) "Exploring time perspective in Greek young adults: validation of the Zimbardo Time Perspective Inventory and relationships with mental health indicators". Social Indicators Research 106, 41-59.

Aluoja, A., J. Shlik, V. Vasar, K. Luuk, and M. Leinsalu (1999) "Development and psychometric properties of the Emotional State Questionnaire, a self-report questionnaire for depression and anxiety". Nordic Journal of Psychiatry 53, 443-449.

Bennet, M. R. and P. M. S. Hacker (2003) Philosophical foundations of neuroscience. Maldan, MA: Blackwell Publishing.

Boniwell, I. (2005). "Beyond time management: how the latest research on time perspective and perceived time use can assist clients with time-related concerns". International Journal of Evidence Based Coaching and Mentoring, 3(2), 61-74.

Boniwell, I., E. Osin, P. A. Linley, and G. V. Ivanchenko (2010) "A question of balance: time perspective and well-being in British and Russian samples". Journal of Positive Psychology $5,24-40$.

Borg, I. and P. J. F. Groenen (2010) Modern Multidimensional Scaling: theory and applications. 2nd ed. (Springer Series in Statistics.) New York, NY: Springer Science + Business Media.

Boyd, J. N and P. Zimbardo (2006) "Constructing time after death: the transcendental future time perspective". In The survival of human consciousness: essays on the possibility of life after death, 107-126. G. L. Lance and M. A. Thalbourne, eds. Jefferson, NC, US: McFarland \& Co.

Brown, K. W. and M. P. Holt (2011) "Experiential processing and the integration of bright and dark sides of the human psyche". In Designing the future of positive psychology: taking the stock and moving forward, 147-159. K. Sheldon, T. Kashdan, and M. Steger, eds. Oxford University Press.

Brown, K. W. and R. M. Ryan (2003) "The benefits of being present: mindfulness and its role in psychological well-being”. Journal of Personality and Social Psychology 84, 822-848.

Brown, K. W. and R. M. Ryan (2004) "Perils and promise in defining and measuring mindfulness: observations from experience". Clinical Psychology: Science \& Practice 11, 242-248.

Burris, C. T. and K. Bailey (2009) "What lies beyond: theory and measurement of after death beliefs?". The International Journal for the Psychology of Religion 19, 173-186.

Carelli, M., B. Wiberg, and M. Wiberg (2011) "Development and construct validation of the Swedish Zimbardo Time Perspective Inventory". European Journal of Psychological Assessment 27, 4, 220-227. 
Clarke, S. M., B. R. Jr. Hayslip, R. Edmondson, and C. A. Guarnaccia (2003) "Religiosity, afterlife beliefs, and bereavement adjustment in adulthood". Journal of Religious Gerontology 14, 207-224.

Crick, F. (1994) The astonishing hypothesis: the scientific search for the soul. New York: Touchtone.

Damasio, A. R. (2001) "Thinking about belief: concluding remarks". In Memory, brain, and belief, 325-335. D. L. Schacter and E. Scarry, eds. Cambridge, MA: Harvard College.

D'Argembeau, A., D. Stawarczyk, S. Majerus, F. Collette, M. Van der Linden, D. Feyers, P. Maquet, and E. Salomon (2010) "The neural basis of personal goal processing when envisioning future events". Journal of Cognitive Neuroscience 22, 1701-13.

Dezutter, J., B. Soenens, K. Luyckx, S. Bruyneel, M. Vansteenkiste, B. Duriez, and D. Hutsebaut (2008) "The role of religion in death attitudes: distinguishing between religious belief and style of processing". Religious Contents, Death Studies 33, 73-92.

Drake, L., E. Duncan, F. Sutherland, C. Abernethy, and C. Henry (2008) "Time perspective and correlates of well-being". Time and Society 17, 47-61.

Elahi, F. (2007) "Death anxiety, afterlife belief, and patients with terminal cancer". Proquest Dissertations and Thesis.

Flannelly, K. J., C. G. Ellison, K. Galek, and H. G. Koenig (2008) "Beliefs about life-after-death, psychiatric symptomology and cognitive theories of psychopathology". Journal of Psychology \& Theology 36, 94-103.

Flannelly, K., H. Koenig, C. Ellison, K. Galek, and N. Krause (2006) "Belief in life after death and mental health: findings from a national survey". The Journal of Nervous and Mental Disease 194, 7, 524-529.

Galek, K. and M. Porter (2010) "A brief review of religious beliefs in research on mental health and ETAS theory". Journal of Health Care Chaplaincy 16, 58-64.

Gawthrop, D. (1999) Vanishing halo: saving the boreal forest. Seattle, Washington: The Mountaineers.

Giovannoli, J. (2000) The biology of belief: how our biology biases our beliefs and perceptions. Rosettapress.com: Rosetta Press.

Giugère, G. (2006) "Collecting and analyzing data in multidimensional scaling experiments: A guide for psychologists using SPSS". Tutorials in Quantitative Methods for Psychology 21, $1,26-37$.

Goldstein, L., A. Brennan, M. Deutsch, and J. Y. Lau (2005) Logic: key concepts in philosophy. New York, NY: Continuum.

Gollnick, J. (2005) Religion and spirituality in the life cycle. New York, NY: Peter Lang.

Hood, R. W. Jr., P. C. Hill, and B. Spilka (2009) The psychology of religion: an empirical approach. 4th ed. New York, NY: Guilford Press.

Kaasik, A. (2004) "Old Estonian religions". Estonian Culture 2, 14-16.

Koening, H. G. (2005) Faith and mental health: religious resources for healing. West Conshohocken, PA: Templeton Foundation Press.

McClain-Jacobson, C., B. Rosenfeld, A. Kosinski, H. Pessin, J. E. Cimino, and W. Breitbart (2004) "Belief in an afterlife, spiritual well-being and end-of-life despair in patients with advanced cancer". General Hospital Psychiatry 26, 484-486.

Nyberg, L, A. Kim, R. Habib, B. Levine, and Tulving, E. (2010) "Consciousness of subjective time in the brain". Proceedings Of The National Academy Of Sciences Of The United States Of America, 107, 51, 22356-22359.

Newberg, A., E. D'Aquili, and V. Rause (2001) Why God won't go away: brain science and the biology of belief. New York, NY: A Ballantine Book.

O'Connor, K. (2006) Culture and customs of the Baltic States. New York, CT: Greenwood Press.

Pullmann, H. and J. Allik (2000) "The Rosenberg Self-Esteem Scale: Its dimensionality, stability, and personality correlates in Estonian”. Personality and Individual Differences 28, 701-715.

Romero, H. R. and J. M. Uomoto (2009) "Time perspective, acculturation, and psychological wellbeing in Mexican Americans". Proquest Dissertations and Theses. 
Rose, B. M. and M. J. O'Sullivan (2002) "Afterlife beliefs and death anxiety: an exploration of the relationship between afterlife expectations and fear of death in an undergraduate population". Omega: Journal of Death and Dying 45, 3, 229-243.

Roseanu, G., M. Marian, I. M. Tomulescu, and C. T. Pusta (2008) "Personal time and psychopathology". Annals of General Psychiatry 7, Suppl. 1.

Rosenberg, M. (1989) Society and the adolescent self-image: revised edition. Middletown, CT: Wesleyan University Press.

Salokangas, R. K., O. Poutanen and E. Stengård (1995) "Screening for depression in primary care. Development and validation of the Depression Scale, a screening instrument for depression". Acta Psychiatrica Scandinavica 92, 10-6.

Schellenberg, J. L. (2005) Prolegomena to philosophy of religion. New York: Cornell University.

Schihalejev, O. (2010) From indifference to dialogue? Estonian young people, the school and religious diversity. (Religious diversity and education in Europe, 19.) New York, NY: Waxmann.

Seema, R., K. W. Brown, A. Sircova, K. Konstabel, J. Quaglia and A. Baltin (submitted) "Validation of Mindful Attention Awareness Scale: adapted in the Estonian with unusual grammar of time".

Seema, R. and A. Sircova (submitted). "Mindfulness - a time perspective? The Estonian study". Baltic Journal of Psychology.

Siegel, D. J. (2012) Developing mind: how relationships and the brain interact to shape who we are. 4th ed. New York, NY: Mind Your Brain.

Sircova, A. and O. V. Mitina (2008) "Vozrastnaja dinamika vremennyx orientacij ličnosti”. Voprosy Psixologii 2, 41-55.

Sircova, A., E. T. Sokolova, and O. V. Mitina (2008) “Adaptacija oprosnika vremennoj perspektivy licnosti F. Zimbardo”. Psixologičesi Žurnal” 29, 101-109.

Sisask, M., A. Värnik, K. Kõlves, K. Konstabel, and D. Wasserman (2008) "Subjective psychological well-being (WHO -5) in assessment of the severity of suicide attempt". Nordic Journal of Psychiatry 62, 431-435.

Suddendorf, T. and M. C. Corballis (2007) "The evolution of foresight: what is mental time travel, and is it unique to humans?". Behavioral and Brain Sciences 30, 299-313.

The 1st International Confernce on Time Perspective (2012) Book of Abstracts. Converging Paths in Psychology Time Theory and Research. University of Coimbra. Portugal. EspacoBranco.

Trull, T. J. and M. J. Prinstein (2013) Clinical psychology. 8th ed. Belmont, CA: Nadsworth Gengage Learning.

Tulving, E. (2002) Mälu. [Memory.] J. Allik, transl. Tartu: Tartu Ülikooli Kirjastus.

Tulving, E. and A. Kim (2007) "The evolution of foresight: what is mental time travel, and is it unique to humans?". Behavioral and Brain Sciences 30, 334-335.

Tulving, E. and K. Szpunar (2012) "Does the future exist?". In Mind and the frontal lobes: cognition, behavior, and brain imaging, 248-264. B. Levine and J. M. Craik, eds. New York, NY: Oxford University Press.

Vandekerckhove, M. and J. Panksepp (2009) "The flow of anoetic to noetic and autonoetic consciousness: a vision of unknowing (anoetic) and knowing (noetic) consciousness in the remembrance of things past and imagined futures". Consciousness and Cognition 18, 10181028.

Vandekerckhove, M. and J. Panksepp (2011) "A neurocognitive theory of higher mental emergence: from anoetic affective experiences to noetic knowledge and autonoetic awareness". Neuroscience and Biobehavioral Reviews 35, 2017-2025.

Villoro, L. (1998) Belief, personal, and propositional knowledge. E. D. Sosa and D. McDermid, transl. Amsterdam-Atlanta, GA: Rodopi.

Westbery, C. and D. C. Dennett (2000) "Mining the past to construe the futuure: Memory, and belief as forms of knowledge". In Memory, brain, and belief, 11-35. D. L. Schacter and E. Scarry, eds. Cambridge, MA: Harvard College. 
Zhang, J. W., and R. T. Howell (2011). "Do time perspectives predict unique variances in life satisfaction beyond personality traits?" Personality and Individual Differences, 50(8), 12611266.

Zimbardo, P. and J. N. Boyd (1999) "Putting time in perspective: a valid, reliable individualdifferences metric". Journal of Personality and Social Psychology 77, 1271-1288.

Zimbardo, P. and J. Boyd (2008) The time paradox: the new psychology of time that will change your life. New York. NY: Free Press.

\section{Web references}

Kairos Future. (2007) Estonian youth in a global context. Available online at $<\mathrm{http}: / /$ www.mynewsdesk.com/se/pressroom/kairosfuture/pressrelease/view/estonian-youth-in-aglobal-context-165919>. Accessed on 13.03.2011.

Annual Report on International Religious Freedom Report (2010). Available online at $<\mathrm{http}: / /$ www.state.gov/j/drl/rls/irf/2010/148930.htm>. Accessed on 12.11.2011.

Ortuño. V. E. C., P. M. Paxão, J. N. Janeiro, and C. V. M. Gomes (2012) Group differences in the Transcendental - Future Time Perspective. Portuguese results. Available online at $<\mathrm{http}: / /$ www.academia.edu/2003294/Group_Differences_in_the_Transcendental-Future_ Time_Perspective_Portuguese_Results $>$. Accessed on 26.11.2012.

Special Eurobarometer 225. (2005) Social values, science \& technology. Available online at $<$ http://ec.europa.eu/public opinion/archives/ebs/ebs 225 report en.pdf $>$. Accessed on 01.05.2012.

Van Deun, K. and L. Delbeke (2000) Multidimensional scaling: open and distance learning. Available online at $<$ http://www.mathpsyc.uni-bonn.de/doc/delbeke/delbeke.htm>. Accessed on 07.11.2012. 
